\title{
Prevalência de Sintomas Depressivos em Estudantes de Medicina com Currículo de Aprendizagem Baseada em Problemas
}

\author{
Prevalence of Depressive Symptoms in Medical Students \\ Following a Problem-Based Learning Curriculum
}

\author{
Heros Aureliano Antunes da Silva Maia ${ }^{I}$ (D) \\ Ana Carolina Silva Assunção ${ }^{I}$ (iD \\ Caroline Santos Silva ${ }^{I}$ (iD \\ Juliana Laranjeira Pereira dos Santos ${ }^{I}(\mathbb{D}$ \\ Carla Jamile Jabar Menezes ${ }^{I}(\mathbb{D}$ \\ José de Bessa Júnior ${ }^{I}(\mathbb{D}$
}

\section{PALAVRAS-CHAVE}

- Aprendizagem Baseada em Problemas.

- Depressão.

- Estudantes de Medicina.

- Educação Médica.

- Saúde Mental.

Introdução: A depressão é um distúrbio heterogêneo, com etiologia, evolução e resposta terapêutica variadas, com relatos de aumento crescente na incidencia entre os jovens. Dois objetivos nortearam este estudo: estimar a prevalência de sintomas depressivos entre acadêmicos de Medicina de uma universidade com métodos ativos de aprendizagem e investigar possíveis associações com variáveis sociodemográficas. Métodos: Trata-se de um estudo transversal descritivo. Aplicaram-se um questionário eletrônico com variáveis sociodemográficas e o Inventário de Depressão de Beck (BDI). Foram realizadas análise univariada e regressão logística multivariada. Resultados: Avaliamos 173 discentes, com discreta predominância de rapazes ( $n=93,53,7 \%) e$ idade mediana de 24 (22-26) anos. Verificaram-se sintomas depressivos em 46,2\% $(n=80)$, dos quais 33,5\% $(n=58)$ leves, $9,2 \%(n=16)$ moderados e 3,4\% $(n=6)$ graves. Sexo feminino $(p=0,032)$ e insatisfação com a Aprendizagem Baseada em Problemas - ABP $(p<0,001)$ se associaram de forma independente aos sintomas depressivos em regressão logística multivariada, com aumento na chance de sintomas depressivos de 2 e 3,5 vezes, respectivamente. Os fatores morar com os pais, ter outros diagnósticos psiquiátricos e praticar regularmente atividade física se associaram aos sintomas depressivos apenas em análise univariada. Conclusão: Os acadêmicos de Medicina apresentaram significativa prevalência de sintomas depressivos. A associação dos sintomas depressivos com insatisfação com o método ABP pode fomentar reflexões sobre a conduta pedagógica $e$ as deficiências na aplicação da metodologia $A B P$ na referida universidade. Ressaltamos a importância da implementação da atividade física no projeto pedagógico e curricular do curso de Medicina como estratégia para a promoção de saúde mental e física nos discentes. 


\section{KEYWORDS}

- Problem-Based Learning.

- Depression.

- Medical Students.

- Medical Education.

- Mental Health.

Recebido em $22 / 6 / 20$

Aceito em 24/6/20

\section{INTRODUCÃO}

A depressão é um distúrbio heterogêneo com curso variável, resposta inconsistente ao tratamento e fisiopatologia multifatorial. A detecção, o diagnóstico e o manejo da depressão são desafiadores para os profissionais de saúde, o que prejudica o funcionamento psicossocial e reduz a qualidade de vida do paciente ${ }^{1}$. Em 2015, a Organização Mundial de Saúde (OMS) estimou em $4,4 \%$ a prevalência global de depressão, o que representou um aumento de $18,4 \%$ em relação a 2005 . Tal agravo é o maior contribuinte individual para incapacidade (7,5\% dos anos vividos com incapacidade) e para suicídio (800 mil casos por ano $)^{2}$.

Entre os mecanismos fisiopatológicos da depressão, destaca-se a hipótese da deficiência de monoaminas, base para o tratamento antidepressivo atual, mas que falha em explicar a variabilidade da apresentação clínica e da resposta aos antidepressivos. Pensa-se ainda em fatores genéticos, alterações no eixo hipotálamo-hipofisário-adrenal, inflamação e distúrbios relacionados à neuroplasticidade, neurogênese e epigenética ${ }^{1,3}$.

Os transtornos depressivos são caracterizados por sintomas como presença de humor triste ou vazio, alterações somáticas e/ou cognitivas e prejuízo na funcionalidade individual ${ }^{4}$. Entre os grupos populacionais vulneráveis, destacam-se os estudantes universitários. Estima-se que $15 \%$ a $25 \%$ desse grupo apresente transtornos psiquiátricos durante sua formação acadêmica, notadamente transtornos depressivos e de ansiedade ${ }^{5}$. Uma metanálise que avaliou especificamente alunos dos cursos médicos apontou uma prevalência média de depressão de $27,2 \%$, com prevalências que variaram de $9,3 \%$ a $55,9 \%{ }^{6}$.

As escolas médicas propiciam um ambiente psicológico tóxico, traduzido por alta carga horária, falta de tempo para lazer, forte competição entre os colegas, exposição à morte e ao sofrimento humanos, situações de abuso, dificuldades financeiras, insegurança em relação ao ingresso no mercado de trabalho, autocobrança e cobrança da sociedade ${ }^{5,7-9}$. A despeito do alto nível de aflição durante o curso de Medicina, os discentes

\section{ABSTRACT}

Introduction: Depression is a heterogenous disorder of diverse etiology, progression and therapeutic response. Increasing incidence of depression in young adulthood has been reported. The purpose of this paper was to evaluate the prevalence of depressive symptoms among medical students at a university which adopts an active learning method and to investigate possible associations to sociodemographic variables. Methods: Descriptive, cross-sectional study. An electronic questionnaire was applied to evaluate sociodemographic variables and depressive symptoms using the Beck Depression Inventory. Univariate and multivariate logistic regression analysis were performed. Results: A slight male predominance $(n=93,53.7 \%)$ was found among 173 students, along with an average median age of 24 [22-26]. Depressive symptoms were identified in $46.2 \%$ of the student $(n=80): 33.5 \%$ ( $n=58)$ with mild symptoms, 9.2\% ( $n=16)$ moderate, and $3.4 \%(n=6)$ severe. Female gender $(p=0.032)$ and dissatisfaction with the active learning method $(p<0.001)$ were independently associated with sysive symptoms in a multivariate logistic regression analysis with the chance of suffering from depressive and lack of regular physical exercise were associated with depressive symptoms only in univariate analysis. Conclusion: The medical students presented a high prevalence rate of depressive symptons. Association between dissatisfaction with the active learning method and depressive symptoms may offer some insight regarding the pedagogical practices and deficiencies in the application of this method at the university in question. It is important to implement strategies that incorporate physical exercise into the pedagogical and curricular project to promote the mental and physical health of the students. 
Os dados foram coletados por meio de aplicação de um questionário anônimo on-line, disponibilizado na plataforma Google Forms. O link do questionário foi enviado ao e-mail de cada estudante e para grupos de WhatsApp. O questionário continha, em primeiro plano, o Termo de Consentimento Livre e Esclarecido (TCLE). Somente após a leitura do TCLE e da seleção da opção "aceito participar da pesquisa", eram disponibilizados o questionário sociodemográfico e o Inventário de Depressão de Beck (Beck Depression Inventory - BDI).

O BDI é um instrumento de autoavaliação com fins de rastreio, sem pretensão diagnóstica ${ }^{12}$, validado em amostras clínicas e populacionais brasileiras ${ }^{13}$ e composto de 21 itens. Cada item avalia um determinado sintoma depressivo presente na última semana, com intensidade de 0 a 3, totalizando um escore de 0 a 63 pontos. $\mathrm{Na}$ análise, consideramos os pontos de corte sugeridos pelo Center for Cognitive Therapy: pontuações de 0 a 9 (pessoa sem sintomas de depressão/sintomas mínimos), de 10 a 18 pontos (sintomas de depressão leve a moderada), de 19 a 29 pontos (sintomas de depressão moderada a grave) e $\geq 30$ pontos (sintomas de depressão grave $)^{14}$.

$\mathrm{Na}$ análise estatística, as variáveis quantitativas, contínuas ou ordinais, foram descritas por suas medidas de tendência central (médias ou medianas) e pelas respectivas medidas de dispersão (desvio padrão, variação interquartil ou valores mínimos e máximos), enquanto as nominais ou qualitativas, por seus valores absolutos e percentagens. $\mathrm{Na}$ comparação dos dados categóricos, utilizaram-se os testes de Fisher ou do qui-quadrado e suas variantes.

Utilizou-se a análise univariada para determinar a associação entre as variáveis sociodemográficas e a prevalência de sintomas depressivos. Calcularam as odds ratio (OR) para cada variável com intervalos de confiança de 95\% (IC95\%). Em seguida, variáveis com valor de $\mathrm{p}<0,1$ na análise univariada foram incluídas em regressão logística multivariada, permanecendo no modelo final se $\mathrm{p}<0,05$. Nas análises, empregou-se o programa estatístico computacional GraphPad Prism, versão 6.0.3, GraphPad Software, San Diego - Califórnia, EUA.

Foram seguidas integralmente as recomendações da Resolução $\mathrm{n}^{\circ}$ 466/2012 do Conselho Nacional de Saúde ${ }^{15}$ a fim de atender aos princípios éticos que envolvem as pesquisas com seres humanos. O projeto foi apreciado e aprovado pelo Comitê de Ética em Pesquisa da Uefs por meio do Parecer no 3.212.213.

Como estratégia para minorar os possíveis danos oriundos desta pesquisa e acolher os alunos participantes que sentissem necessidade, simultânea à manutenção de seu anonimato, informaram-se, no início e no final do questionário on-line, o local de funcionamento e a disponibilidade (horário de funcionamento, telefone e e-mail) dos serviços prestados pela Comissão de Apoio ao Discente do Colegiado de Medicina e pelo Núcleo de Apoio Psicossocial e Pedagógico da Uefs, os quais englobam profissionais da psiquiatria, pedagogia, psicopedagogia, psicologia e assistência social.

\section{RESULTADOS}

No ano de realização do estudo, havia 191 alunos matriculados no curso de Medicina da Uefs, com predominância do sexo masculino (55,5\%). Obtivemos participação de 173 acadêmicos, o que representou uma taxa de resposta de $90,5 \%$ e manutenção do predomínio do sexo masculino: 93 homens (53,7\%). A idade mediana dos participantes foi de
24 (22-26) anos. Dos participantes, 66 (38,1\%) estavam no internato médico, $73(42,2 \%)$ moravam com os pais, $92(53,2 \%)$ referiram ter parceiro fixo, $73(42,2 \%)$ tinham atividade extracurricular remunerada, $33(19,1 \%)$ não estavam satisfeitos com o método ABP, $112(64,7 \%)$ praticavam atividade física e $92(53,2 \%)$ relataram uso de álcool (Tabela 1$)$.

Diagnóstico prévio ou atual de depressão foi relatado por 17 (9,8\%) participantes, $23(13,3 \%)$ já realizaram tratamento psicológico e 12 (6,9\%) fizeram tratamento medicamentoso. Ansiedade foi o transtorno relatado mais frequentemente associado nessa população: $41(23,7 \%)$ dos participantes. Outros transtornos foram referidos por 6,9\% dos alunos: transtorno do déficit de atenção com hiperatividade (três $=1,7 \%$ ), síndrome do pânico (três $=1,7 \%$ ), transtorno bipolar (dois $=1,1 \%$ ), transtorno obsessivo-compulsivo (dois $=1,1 \%$ ), transtorno alimentar (um $=0,5 \%)$ e fobia social $(\mathrm{um}=0,5 \%)$.

\begin{tabular}{|c|c|c|c|c|c|c|}
\hline Caracterí & $\begin{array}{l}\text { cas soc } \\
\text { preser }\end{array}$ & $\begin{array}{l}\text { emo } \\
\text { de si }\end{array}$ & $\begin{array}{l}\text { a } 1 \\
\text { cas do } \\
\text { as de }\end{array}$ & $\begin{array}{l}\text { estuda } \\
\text { epress }\end{array}$ & segund & \\
\hline Variáveis & Total & De & dos & Não c & imidos & $\mathbf{P}$ \\
\hline & n & $\mathbf{n}$ & $\%$ & $\mathbf{n}$ & $\%$ & \\
\hline Sexo & & & & & & \\
\hline Feminino & 80 & 46 & 57,5 & 34 & 36,6 & 0,006 \\
\hline Masculino & 93 & 34 & 42,5 & 59 & 63,4 & \\
\hline Série & & & & & & \\
\hline Do $1^{\circ}$ ao $4^{\circ}$ ano & 107 & 53 & 66,2 & 54 & 58,1 & 0,277 \\
\hline Do $5^{\circ}$ ao $6^{\circ}$ ano & 66 & 27 & 33,8 & 39 & 41,9 & \\
\hline Mora com os pa & & & & & & \\
\hline Sim & 73 & 40 & 50 & 33 & 35,5 & 0,053 \\
\hline Não & 100 & 40 & 50 & 60 & 64,5 & \\
\hline Parceiro fixo & & & & & & \\
\hline Sim & 92 & 40 & 50 & 52 & 55,9 & 0,449 \\
\hline Não & 81 & 40 & 50 & 41 & 44,1 & \\
\hline Atividade física & & & & & & \\
\hline Sim & 112 & 45 & 56,2 & 67 & 72,1 & 0,038 \\
\hline Não & 61 & 35 & 43,8 & 26 & 27,9 & \\
\hline Insatisfação com & $\mathrm{ABP}$ & & & & & \\
\hline Sim & 33 & 24 & 30 & 9 & 9,7 & $<0,001$ \\
\hline Não & 140 & 56 & 70 & 84 & 90,3 & \\
\hline Atividade extrac & ricular & uner & & & & \\
\hline Sim & 77 & 35 & 43,8 & 38 & 40,9 & 0,758 \\
\hline Não & 100 & 45 & 56,2 & 55 & 59,1 & \\
\hline Uso de álcool & & & & & & \\
\hline Sim & 94 & 39 & 48,8 & 55 & 59,1 & 0,554 \\
\hline Não & 79 & 41 & 51,2 & 38 & 40,9 & \\
\hline Outros diagnóst & s psiqu & icos & & & & \\
\hline Sim & 51 & 30 & 37,5 & 21 & 22,6 & 0,044 \\
\hline Não & 122 & 50 & 62,5 & 72 & 77,4 & \\
\hline
\end{tabular}

Fonte: Elaborada pelos autores.

\begin{tabular}{l|l} 
REVISTA BRASILEIRA DE EDUCAÇÃO MÉDICA \\
\hline $44(3)$ : e105; 2020
\end{tabular} 


\begin{tabular}{|c|c|c|c|c|c|c|c|c|}
\hline \multirow{3}{*}{ Variáveis } & \multicolumn{8}{|c|}{ Sintomas depressivos } \\
\hline & \multicolumn{2}{|c|}{$\begin{array}{l}\text { Sem sintomas } \\
\text { (0-9 pontos) }\end{array}$} & \multicolumn{2}{|c|}{$\begin{array}{c}\text { Leves } \\
(10-18 \text { pontos })\end{array}$} & \multicolumn{2}{|c|}{$\begin{array}{c}\text { Moderados } \\
(19-29 \text { pontos })\end{array}$} & \multicolumn{2}{|c|}{$\begin{array}{c}\text { Graves } \\
(\geq 30 \text { pontos })\end{array}$} \\
\hline & $\mathbf{n}$ & $\%$ & $\mathbf{n}$ & $\%$ & $\mathbf{n}$ & $\%$ & $\mathbf{n}$ & $\%$ \\
\hline \multicolumn{9}{|l|}{ Série do curso } \\
\hline $1^{\circ}$ ano $(n=32)$ & 16 & $50 \%$ & 11 & $34,4 \%$ & 4 & $12,5 \%$ & 1 & $3,1 \%$ \\
\hline $2^{\circ}$ ano $(n=25)$ & 15 & $60 \%$ & 7 & $28 \%$ & 2 & $8 \%$ & 1 & $4 \%$ \\
\hline $3^{\circ}$ ano $(n=30)$ & 17 & $56,7 \%$ & 11 & $36,7 \%$ & 2 & $6,6 \%$ & 0 & $0 \%$ \\
\hline $4^{\circ}$ ano $(n=20)$ & 6 & $30 \%$ & 7 & $35 \%$ & 6 & $30 \%$ & 1 & $5 \%$ \\
\hline $5^{\circ}$ ano $(n=34)$ & 19 & $55,9 \%$ & 11 & $32,3 \%$ & 2 & $5,9 \%$ & 2 & $5,9 \%$ \\
\hline $6^{\circ}$ ano $(n=32)$ & 20 & $62,5 \%$ & 11 & $34,4 \%$ & 0 & $0 \%$ & 1 & $3,1 \%$ \\
\hline Total $(n=173)$ & 93 & $53,8 \%$ & 58 & $33,5 \%$ & 16 & $9,2 \%$ & 6 & $3,4 \%$ \\
\hline
\end{tabular}

Fonte: Elaborada pelos autores.

De acordo com os escores do BDI adotados, observou-se a prevalência de sintomas depressivos em 80 (46,2\%) participantes. Desses sintomas, $58(33,5 \%)$ foram classificados como leves, $16(9,2 \%)$ como moderados e $6(3,4 \%)$ como graves. A distribuição da prevalência de sintomas depressivos para cada série do curso é apontada na Tabela 2. Autoacusações $(75,1 \%)$, fadiga $(74 \%)$ e distúrbios do sono $(65,3 \%)$ foram os sintomas depressivos mais prevalentes. Ideação suicida foi referida por $9,8 \%$; a frequência de cada sintoma está mais bem detalhada na Tabela 3 .

As características sociodemográficas dos discentes que se associaram com a presença de sintomas de depressão na análise univariada (Tabela 4) foram prática de atividades físicas $(\mathrm{OR}=0,49$ [0,26-0,95] IC95\%) $(\mathrm{p}=0,038)$, sexo feminino $(\mathrm{OR}=2,34[1,27-$ $4,32] \mathrm{IC} 95 \%)(\mathrm{p}=0,006)$, morar com os pais $(\mathrm{OR}=1,8[0,98-3,34]$ IC95\% $(\mathrm{p}=0,053)$, outros diagnósticos psiquiátricos $(\mathrm{OR}=2,05$ $[1,05-3,96]$ IC95\%) $(\mathrm{p}=0,044)$ e insatisfação com a ABP $(\mathrm{OR}=4$ $[1,72-9,09]$ IC95\%) $(\mathrm{p}<0,001)$.

Após a regressão logística multivariada (Tabela 4), apenas sexo feminino e insatisfação com a ABP permaneceram no modelo final como variáveis independentes associadas aos sintomas depressivos. Sexo feminino $(\mathrm{p}=0,032)$ e estar insatisfeito com a metodologia ABP $(\mathrm{p}<0,001)$ aumentam a chance de sintomas depressivos em 2,05 e 3,57 vezes, respectivamente.

Não foram evidenciadas diferenças estatisticamente significantes na prevalência de sintomas depressivos relacionadas ao ciclo do curso, à estabilidade dos relacionamentos afetivos, à atividade extracurricular remunerada e ao uso de bebidas alcoólicas.

\begin{tabular}{|c|c|c|c|}
\hline \multicolumn{4}{|c|}{$\begin{array}{l}\text { Prevalência de cada sintoma depressivo avaliado pelo Inventário de } \\
\text { Depressão de Beck }\end{array}$} \\
\hline Sintomas depressivos & Questão & $\mathbf{N}$ & $\%$ \\
\hline Autoacusações & 8 & 130 & $75,1 \%$ \\
\hline Fadiga & 17 & 128 & $74 \%$ \\
\hline Distúrbios do sono & 16 & 113 & $65,3 \%$ \\
\hline Irritabilidade & 11 & 101 & $58,4 \%$ \\
\hline Indecisão & 13 & 85 & $49,1 \%$ \\
\hline Falta de satisfação & 4 & 84 & $48,5 \%$ \\
\hline Inibição para o trabalho & 15 & 84 & $48,5 \%$ \\
\hline Autodepreciação & 7 & 81 & $46,8 \%$ \\
\hline Retração social & 12 & 78 & $45,1 \%$ \\
\hline Preocupação somática & 20 & 77 & $44,5 \%$ \\
\hline Sensação de culpa & 5 & 74 & $42,7 \%$ \\
\hline Distorção da imagem corporal & 14 & 67 & $38,7 \%$ \\
\hline Tristeza & 1 & 66 & $38,1 \%$ \\
\hline Crises de choro & 10 & 54 & $31,2 \%$ \\
\hline Pessimismo & 2 & 49 & $28,3 \%$ \\
\hline Redução da libido & 21 & 49 & $28,3 \%$ \\
\hline Sensação de fracasso & 3 & 47 & $27,2 \%$ \\
\hline Perda de apetite & 18 & 45 & $26 \%$ \\
\hline Sensação de punição & 6 & 44 & $25,4 \%$ \\
\hline Perda de peso & 19 & 33 & $19,1 \%$ \\
\hline Ideias suicidas & 9 & 17 & $9,8 \%$ \\
\hline
\end{tabular}

Fonte: Elaborada pelos autores. 


\begin{tabular}{|c|c|c|c|c|c|c|}
\hline \multirow{2}{*}{ Variáveis } & \multicolumn{3}{|c|}{ Análise univariada } & \multicolumn{3}{|c|}{ Análise multivariada } \\
\hline & OR & IC95\% & $\mathbf{p}$ & OR & IC95\% & $\mathbf{p}$ \\
\hline Sexo feminino & 2,34 & $1,27-4,32$ & 0,006 & 2,05 & $1,06-3,96$ & 0,032 \\
\hline Série do curso & 1,41 & $0,74-2,72$ & 0,277 & - & - & - \\
\hline Morar com os pais & 1,8 & $0,98-3,34$ & 0,053 & 1,94 & $0,99-3,78$ & 0,051 \\
\hline Parceiro fixo & 0,79 & $0,42-1,44$ & 0,449 & - & - & - \\
\hline Atividade física & 0,49 & $0,26-0,95$ & 0,038 & 0,61 & $0,33-1,22$ & 0,16 \\
\hline Insatisfação com a $\mathrm{ABP}$ & 4 & $1,72-9,09$ & $<0,001$ & 3,57 & $1,44-8,33$ & $<0,001$ \\
\hline $\begin{array}{l}\text { Atividade extracurricular } \\
\text { remunerada }\end{array}$ & 1,12 & $0,61-2,01$ & 0,758 & - & - & - \\
\hline Uso de álcool & 0,66 & $0,36-1,21$ & 0,554 & - & - & - \\
\hline Outros diagnósticos psiquiátricos & 2,05 & $1,05-3,96$ & 0,044 & 1,81 & $0,88-3,74$ & 0,105 \\
\hline
\end{tabular}

Fonte: Elaborada pelos autores.

\section{DISCUSSÃO}

A depressão é mais comum entre estudantes de Medicina que entre a população em geral $^{16}$. Em comparação a estudos realizados com metodologia semelhante (mesmos pontos de corte para o BDI), a prevalência de sintomas depressivos encontrada no curso pesquisado foi similar à encontrada em outras universidades do Brasil $(41,3 \%)^{9}$. A prevalência também foi similar na Índia $(48,4 \%)^{17}$, mas inferior na Polônia $(56,3 \%)$ e superior na Alemanha $(34,9 \%)$ e em Portugal $(26 \%)^{18}$.

Não houve diferenças significantes na prevalência de sintomatologia depressiva entre os ciclos ou por série do curso. Contudo, a literatura aponta aumento de sintomatologia depressiva no início do curso, quando há a vivência de uma "fase de decepção" após um deslumbramento inicial, e no anos finais (internato médico), fenômeno atribuído pelos autores à maior privação da vida social, ao contato com a doença e morte, e à dificuldade em comunicação de más notícias ${ }^{7,19,20}$.

A prevalência de sintomas depressivos foi significativamente maior em mulheres, o que é corroborado pela literatura na população em geral e em universitários de Medicina de outras partes do mundo ${ }^{1,4,6,9,16}$. Maiores níveis de marcadores inflamatórios, neurotróficos e serotoninérgicos ${ }^{21}$, diferenças no perfil hormona ${ }^{22}$ e fatores socioculturais como desigualdade de gênero ${ }^{23}$ podem explicar a maior sintomatologia depressiva em mulheres.

Tal cenário se associa com pior qualidade de vida entre as médicas residentes ${ }^{24}$. A despeito de atualmente serem maioria nas escolas de Medicina, as mulheres médicas ainda detêm menor remuneração ${ }^{25}$, são influenciadas pelo "sexismo" em ambientes de aprendizado para escolha de especialidade ${ }^{26}$ e possuem progressão de carreira acadêmica mais lenta ${ }^{25}$. Ainda assim, as mulheres médicas são mais empáticas e seus pacientes têm melhores desfechos quando comparados com os de homens ${ }^{25}$.

$\mathrm{O}$ fato de morar com os pais se associou aos sintomas depressivos. O ambiente familiar é geralmente tido como fator de proteção ${ }^{27}$, e é referido que se afastar do núcleo familiar torna os universitários mais expostos a distúrbios psicológicos ${ }^{5}$. Em contrapartida, a alta expectativa dos pais é tida como fator estressor pelos acadêmicos de Medicina ${ }^{28}$, e a pressão parental já foi associada com maior prevalência de depressão ${ }^{27}$ nesse grupo. Não obstante isso, os discentes que vivem com os pais podem ter menor desempenho acadêmico por conta do comprometimento de seu tempo de estudo pelo ambiente e por causa das atividades familiares ${ }^{29}$.

Constatamos que os alunos não satisfeitos com a $A B P$ têm maior chance de desenvolver sintomas depressivos. Na mesma direção, o curso de Medicina que adotou o método ABP obteve maior prevalência de sintomas depressivos ao ser comparado com curso de método tradicional ${ }^{30}$. $\mathrm{Na}$ metodologia ativa $\mathrm{ABP}$, os acadêmicos são responsáveis pelo próprio aprendizado ao mesmo tempo que são expostos a situações reais de forma precoce. No método tradicional, o conteúdo é transmitido de forma passiva pelo professor, pois o aprendizado é apoiado na memorização por rotina ${ }^{30}$.

A maior prevalência de sintomas depressivos em cursos com ABP pode se relacionar ao maior comprometimento pessoal exigido pelo método e a uma insegurança inicial na organização do aprendizado na transição entre o ensino médio - no método tradicional - e o ingresso no curso de Medicina com $\mathrm{ABP}^{31,32}$. Ademais, deficiências na aplicação do método, como a falta de clareza sobre os limites dos objetivos de aprendizado relatada por "calouros" e a ausência de feedback dos professores na $\mathrm{ABP}$, podem atuar como fatores estressantes ${ }^{33}$.

Os alunos da $\mathrm{ABP}$ ainda relatam uma insegurança em relação à profundidade de aprendizado dos conteúdos, ao real domínio dos conceitos importantes e necessários, ao detalhamento excessivo de temas aparentemente irrelevantes e à quantidade de tempo necessária para estudo. Tais aspectos culminam no temor em falhar em futuras avaliações ${ }^{31}$.

O uso de álcool é frequente entre discentes de Medicina ${ }^{34}$. Atribui-se ao uso excessivo de álcool um modo de escapar das preocupações diárias. Esse hábito é prejudicial e preocupante, visto que os estudantes experimentam mais eventos negativos (blackouts) do que alívio das dificuldades. O uso excessivo de álcool também está associado a comportamento agressivo, direção alcoolizada, relações sexuais desprotegidas e comportamentos sabidamente danosos ${ }^{34}$.

A prevalência de sintomas depressivos foi maior naqueles que referiram outros diagnósticos psiquiátricos. No curso de medicina da Uefs, a ansiedade foi o transtorno mais relatado, com prevalência pouco

\begin{tabular}{l|l}
5 & REVISTA BRASILEIRA DE EDUCAÇÃO MÉDICA \\
\hline 44 (3) : e105; 2020
\end{tabular} 
inferior à encontrada em metanálise de estudos brasileiros $(32,9 \%)^{16}$. A associação entre ansiedade e sintomatologia depressiva em universitários de medicina também já foi descrita na literatura ${ }^{9,35}$. Os transtornos depressivos ainda se associam a transtornos relacionados a substâncias, síndrome do pânico, transtorno obsessivo-compulsivo, anorexia e bulimia nervosas e transtorno de personalidade borderline $e^{4}$.

Dentre os sintomas depressivos avaliados pelo BDI, ideação suicida foi o sintoma menos prevalente entre os participantes deste estudo, o que, de modo algum, não minora o problema nem atenua nossa preocupação. A taxa de suicídio entre acadêmicos de Medicina é maior que na população em geral e em outros grupos de acadêmicos ${ }^{36}$.

A prática de exercícios físicos se associou com menor prevalência de sintomas depressivos no presente estudo. Além de reduzir a sintomatologia depressiva, o exercício físico melhora a qualidade do sono e a função $\operatorname{cognitiva}^{37}$, sendo efetivo como terapia complementar para a depressão ${ }^{38}$. A atividade física está incluída nos guidelines para tratamento da depressão de países como Suécia e Canadá ${ }^{39}$. No curso de Medicina da Uefs, os alunos se organizam para a prática de atividade física e lazer por meio da Associação Atlética de Medicina, a qual participa de campeonatos e confraternizações como o Intermed, competição que reúne os discentes de Medicina dos cursos do estado, momento em que os alunos participantes são dispensados das atividades acadêmicas. Ratificamos a importância da atividade física e sugerimos a implantação de programas de atividade física e prática esportiva regular com objetivo de prevenir/minimizar os sintomas depressivos nessa população.

Os achados desta pesquisa contribuem para alertar sobre a necessidade de reflexão acerca do tema e de planejamento de ações que contribuam para a saúde mental de estudantes de cursos de graduação que trabalham com metodologias inovadoras como a ABP. Muitos são os artigos que apresentam resultados sobre temas como depressão, suicídio e uso de álcool e drogas em discentes de Medicina, todavia, com a difusão das metodologias inovadoras nos cursos médicos, faz-se necessário compreender como elas colaboram ou não para a saúde mental dos alunos.

Entre as limitações deste estudo, ressaltamos seu desenho transversal e sua característica unicêntrica, o que reduz a validade externa. Acreditamos que os resultados não traduzem uma realidade universal, mas certamente podem ser úteis para o planejamento de políticas locais. A despeito dessa limitação, alguns dos nossos achados vão ao encontro da maioria dos artigos sobre a temática, reiterando a gravidade do problema.

Destaca-se a boa impressão do uso de questionários eletrônicos em estudos sobre temas sensíveis em populações de boa compreensão e escolaridade. Entendemos que se minimizam os constrangimentos proporcionados pelas entrevistas presenciais e se proporciona melhor anonimato.

\section{CONCLUSÃO}

O presente estudo revelou que alunos de Medicina da Uefs apresentam alta prevalência de sintomas depressivos, tal qual a encontrada em outras instituições brasileiras e internacionais. Demonstrou-se maior prevalência dos sintomas depressivos nas mulheres, nos que moram com os pais, naqueles com outros diagnósticos psiquiátricos, nos insatisfeitos com a $\mathrm{ABP}$ e naqueles sem atividade física regular; destes, apenas sexo feminino e insatisfação com a ABP se associaram de forma independente aos sintomas depressivos. Vislumbramos que tais achados possam contribuir para a ampliação das ações desenvolvidas pela Comissão de Apoio ao
Discente do Colegiado de Medicina.

A associação entre insatisfação com a $\mathrm{ABP}$ e sintomas depressivos pode suscitar ao curso reflexões sobre as condutas pedagógicas e a aplicação da metodologia ABP. Ressaltamos a importância de se considerar a implementação de atividades esportivas no projeto pedagógico e curricular como estratégia para a promoção da saúde física e mental dos acadêmicos. Talvez resida no apoio às Associações Atléticas de Medicina e no estabelecimento de horários protegidos para atividade física uma opção para a atenuação dos sintomas depressivos nos discentes de Medicina. Reiteramos a necessidade de novos estudos em cursos médicos com metodologia ativa de aprendizagem.

\section{REFERÊNCIAS}

1. Malhi GS, Mann JJ. Depression. Lancet. 2018;392(10161):2299-312.

2. World Health Organization. Depression and other common mental disorders: global health estimates. Geneva: World Health Organization; 2017.

3. Belmaker RH, Agam G. Major depressive disorder. N Engl J Med. 2008 Jan 3;358(1):55-68.

4. American Psychiatric Association. Diagnostic and statistical manual of mental disorders: DSM-V. Arlington, VA: American Psychiatric Association; 2013.

5. Vasconcelos TC, Dias BRT, Andrade LR, Melo GF, Barbosa L, Souza E. Prevalência de sintomas de ansiedade e depressão em estudantes de Medicina. Rev Bras Educ Med. 2015;39(1):135-42.

6. Rotenstein LS, Ramos MA, Torre M, Segal JB, Peluso MJ, Guille C, et al. Prevalence of depression, depressive symptoms, and suicidal ideation among medical students. JAMA. 2016 Dec 6;316(21):2214-36.

7. Cybulski CA, Mansani FP. Análise da depressão, dos fatores de risco para sintomas depressivos e do uso de antidepressivos entre acadêmicos do curso de Medicina da Universidade Estadual de Ponta Grossa. Rev Bras Educ Med. 2017;41(1):92-101.

8. Dyrbye LN, Thomas MR, Shanafelt TD. Medical student distress: causes, consequences, and proposed solutions. Mayo Clin Proc. 2005;80(12):1613-22.

9. Mayer FB, Souza IS, Silveira PSP, Itaqui MHL, Souza ARND, Campos EP, et al. Factors associated to depression and anxiety in medical students: a multicenter study. BMC Med Educ. 2016 Dec 26;16(1):282.

10. Camp DL, Hollingsworth MA, Zaccaro DJ, Cariaga-Lo LD, Richards BF. Does a problem-based learning curriculum affect depression in medical students? Acad Med. 1994;69(10):S25-7.

11. Noronha Júnior MAG, Braga YA, Marques TG, Silva RT, Vieira SD, Coelho VAF, et al. Depression in medical students. Rev Méd Minas Gerais. 2015;25(4):562-7.

12. Costa EFO, Santana YS, Santos ATRA, Martins LAN, Melo EV, Andrade TM. Sintomas depressivos entre internos de medicina em uma universidade pública brasileira. Rev Assoc Med Bras. 2012;58(1):53-9.

13. Gorenstein C, Andrade L. Inventário de Depressão de Beck: propriedades psicométricas da versão em português. Rev Psiquiatr Clín. 1998;25(5):245-50.

14. Beck AT, Steer RA, Carbin MG. Psychometric properties of the Beck Depression Inventory: twenty-five years of evaluation. Clin Psychol Rev. 1988;8(1):77-100. 
15. BRASIL. Conselho Nacional de Saúde. Resolução $n^{\circ}$ 466, de 12 de dezembro de 2012. Aprova normas regulamentadoras de pesquisas envolvendo seres humanos. Diário Oficial da União, Brasília; 2013.

16. Pacheco JP, Giacomin HT, Tam WW, Ribeiro TB, Arab C, Bezerra IM, et al. Mental health problems among medical students in Brazil: a systematic review and meta-analysis. Rev Bras Psiquiatr. 2017 Aug 31;39(4):369-78.

17. Kumar SG, Kattimani S, Sarkar S, Kar S. Prevalence of depression and its relation to stress level among medical students in Puducherry, India. Ind Psychiatry J. 2017;26(1):86-90.

18. Seweryn M, Tyrała K, Kolarczyk-Haczyk A, Bonk M, Bulska W, Krysta K. Evaluation of the level of depression among medical students from Poland, Portugal and Germany. Psychiatr Danub. 2015;27:216-22.

19. Rezende CHA, Abrão CB, Coelho EP, Passos LBS. Prevalência de sintomas depressivos entre estudantes de Medicina da Universidade Federal de Uberlândia. Rev Bras Educ Med. 2008;32(3):315-23.

20. Serra RD, Dinato SLM, Caseiro MM. Prevalência de sintomas depressivos e ansiosos em alunos de Medicina na cidade de Santos. J Bras Psiquiatr. 2015;64(3):213-20.

21. Labaka A, Goñi-Balentziaga O, Lebeña A, Pérez-Tejada J. Biological sex differences in depression: a systematic review. Biol Res Nurs. 2018;20(4):383-92.

22. Zender R, Olshansky E. Women's mental health: depression and anxiety. Nurs Clin North Am. 2009;44(3):355-64.

23. Pacheco JPG, Silveira JB, Ferreira RPC, Lo K, Schineider JR, Giacomin HTA, et al. Gender inequality and depression among medical students: a global meta-regression analysis. J Psychiatr Res. 2019;111:36-43.

24. Kobayasi R, Tempski PZ, Arantes-Costa FM, Martins MA. Gender differences in the perception of quality of life during internal medicine training: a qualitative and quantitative analysis. BMC Med Educ. 2018;18(1):1-14.

25. Bleakley A. Gender matters in medical education. Med Educ. 2013;47(1):59-70.

26. Kristoffersson E, Diderichsen S, Verdonk P, Lagro-Janssen T, Hamberg $\mathrm{K}$, Andersson J. To select or be selected: gendered experiences in clinical training affect medical students' specialty preferences. BMC Med Educ. 2018;18(1):1-11.

27. Tabalipa FO, Souza MF, Pfützenreuter G, Lima VC, Traebert E, Traebert J. Prevalence of anxiety and depression among medical students. Rev Bras Educ Med. 2015;39(3):388-94.

28. Sreeramareddy CT, Shankar PR, Binu VS, Mukhopadhyay C, Ray B, Menezes RG. Psychological morbidity, sources of stress and coping strategies among undergraduate medical students of Nepal. BMC Med Educ. 2007;7:1-8.

29. Fares J, Al Tabosh H, Saadeddin Z, El Mouhayyar C, Aridi H. Stress, burnout and coping strategies in preclinical medical students. North Am J Med Sci. 2016;8(2):75-81.

30. Aragão JA, Freire MRM, Nolasco LGF, Diniz SS, Sant'anna FMA, Sant'anna ICA, et al. Prevalence of depressive symptoms among medical students taught using problem-based learning versus traditional methods. Int J Psychiatry Clin Pract. 2018;22(2):123-8.

31. Lewis AD, Menezes DAB, McDermott HE, Hibbert LJ, Brennan SL, Ross EE, et al. A comparison of course-related stressors in undergraduate problem-based learning (PBL) versus non-PBL medical programmes. BMC Med Educ. 2009;9(1):1-8.

32. Smolka MLRM, Gomes AP, Siqueira-Batista R. Autonomia no contexto pedagógico: percepção de estudantes de Medicina acerca da aprendizagem baseada em problemas. Rev Bras Educ Med. 2014;38(1):5-14

33. Dagistani A, Al Hejaili F, Binsalih S, Al Jahdali H, Al Sayyari A. Stress in medical students in a Problem-Based Learning curriculum. Int J High Educ. 2016;5(3):12-19.

34. Duroy D, Iglesias P, Perquier F, Brahim N, Lejoyeux M. Alcoolisation à risque chez des étudiants en médecine parisiens. Encephale. 2017;43(4):334-9.

35. Fawzy M, Hamed SA. Prevalence of psychological stress, depression and anxiety among medical students in Egypt. Psychiatry Res. 2017;255:186-94.

36. Santa ND, Cantilino A. Suicídio entre médicos e estudantes de Medicina: revisão de literatura. Rev Bras Educ Med. 2016;40(4):772-80.

37. Gourgouvelis J, Yielder P, Clarke ST, Behbahani H, Murphy BA. Exercise leads to better clinical outcomes in those receiving medication plus cognitive behavioral therapy for major depressive disorder. Front Psychiatry. 2018;9:1-12.

38. Mota-Pereira J, Silverio J, Carvalho S, Ribeiro JC, Fonte D, Ramos J. Moderate exercise improves depression parameters in treatmentresistant patients with major depressive disorder. J Psychiatr Res. 2011;45(8):1005-11.

39. Carneiro LF, Mota MP, Schuch F, Deslandes A, Vasconcelos-Raposo J. Portuguese and Brazilian guidelines for the treatment of depression: exercise as medicine. Rev Bras Psiquiatr. 2017 Aug 30;40(2):210-1.

\section{CONTRIBUIÇÃO DOS AUTORES}

Heros Aureliano Antunes da Silva Maia participou do planejamento da pesquisa, revisão bibliográfica, da coleta de dados, da tabulação, da análise estatística de dados, criação de tabelas e redação do texto.

Ana Carolina Silva Assunção participou do planejamento da pesquisa e da coleta de dados.

Caroline Santos Silva participou do planejamento da pesquisa, da coleta de dados e da análise estatística de dados.

Juliana Laranjeira Pereira dos Santos participou do planejamento da pesquisa e revisão crítica da versão final.

Carla Jamile Jabar Menezes participou do planejamento da pesquisa e revisão crítica da versão final.

José de Bessa Júnior orientou e supervisionou a pesquisa, apoiou a elaboração do estudo, orientou a análise estatística de dados, participou da criação de tabelas e redação do texto.

\section{CONFLITO DE INTERESSES}

Os autores declaram não haver conflitos de interesse neste estudo.

\section{ENDEREÇO PARA CORRESPONDÊNCIA}

Heros Aureliano Antunes da Silva Maia. Rua Tijuca, 1160, Parque Ipê, Feira de Santana, BA, Brasil. CEP: 44054-249.

E-mail: herosmaia@hotmail.com 